\title{
Intuitive Interactive Platform for Preoperative Communication Between Hospital and Patients/Caregivers: Towards Community Partnership for Peri-Operative Person-Based Healthcare Model
}

Hwan Ing Hee ${ }^{1^{*}}$, BAO, FAMS, FRCA, MBBch; Jacob Chen ${ }^{2 *}$; Simon Lui ${ }^{2 *}$, PhD

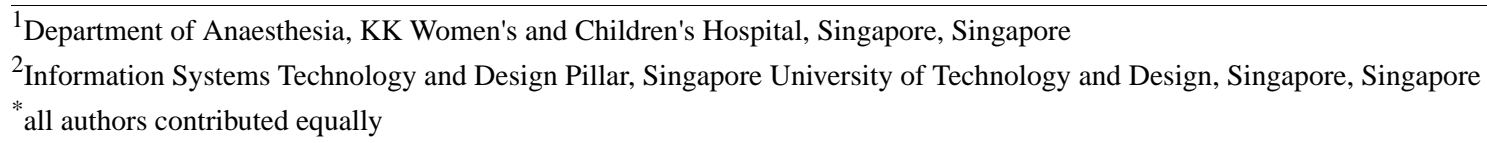

Corresponding Author:

Simon Lui, PhD

Information Systems Technology and Design Pillar

Singapore University of Technology and Design

8 Somapah Road, 1.502-18

Singapore, 487372

Singapore

Phone: 6590274092

Email: simon lui@ sutd.edu.sg

\begin{abstract}
Background: Preoperative phone screening of patients' recent health status and communication to patients about time of admission, surgery and fasting is the back bone of preoperative care in day surgery. Nursing staff are increasingly burdened by non-direct administrative duties, removing them from direct patient care. The amount of time nurses spend on direct patient care have been reported to be less than $40 \%$ of their working hours. This negatively impacts the quality of care, patient outcome and experience, results in job dissatisfaction and burnout among nurses. In our institution, day surgery constituted $50 \%$ of our surgical workload with an estimated 3000 cases per annum.
\end{abstract}

Objective: The objective is to develop a novel application (APP), "Image and Go" (InG), an efficient and secured closed loop system of automated electronic preoperative communication for scheduling day surgery patients. The system frees the nurses from mundane jobs of telephone screening and delivery of routine preoperative instructions and the system is enabled to highlight patients who require a follow-up phone consult for counseling enhancement. InG has minimal intrusion to the current workflow of the nurses. It does not require complicated integration with the hospital's IT system. InG provides real-time overview of the patient's response to the nurses. Nurses can adjust the instructions in a few clicks, the InG backend system automatically reschedules the affected patients. InG is a secure and convenient platform, which taps on SMS/Telegram that millions of people use daily.

Methods: We leveraged the widespread use of printed day surgery schedules in day surgery centers. We developed the InG app for iOS/Android smartphone/tablet. InG takes photo(s) of printed day surgery form(s), identifies the patients' information and carries out interactive preoperative communication with patients via SMS/telegram automatically. 1. A survey was conducted on frontline nurses directly involved in nurse-led preoperative screening and instructions to identify problems and time expenditure during the work process. 2 . We evaluated the performance of InG in a mock test with 20 university students as mock patients. In the test, 12 confirmed the appointment, 6 requested for time change, and 2 requested follow-up phone calls. Then we compared the result with the traditional approach.

Results: 1 . An average of 4 hours was spent in nurse-led phone calls per day. There was strong agreement among $87.5 \%$ nurses that direct patient care gave them job satisfaction, and 75\% strongly agreed that it is more productive to channel time from nurse-led calling to direct patient care. Of those queried, $87.5 \%$ strongly agreed that automation with close loop increases the efficiency of the work process. 2. InG is 6.03 times faster than the traditional approach. InG took 32 minutes to complete the communication with 20 patients. The nurse could work on other duties while InG was processing the telegram communication. The traditional approach took 3 hours 13 min with 20 patients.

Conclusions: The closed loop system of automated electronic preoperative communication provides a secure and efficient communication platform for the hospital-community partnership in healthcare with better utilization of nursing time for direct patient care and greater work satisfaction. 
(iproc 2017;3(1):e12) doi: 10.2196/iproc.8425

\section{KEYWORDS}

preoperative communication; preoperative instructions; telehealth; customer relationship management; optical character recognition; information retrieval; patient screening; automated messaging; automated interaction

\section{Multimedia Appendix 1}

Full poster.

[PDF File (Adobe PDF File), 1MB-Multimedia Appendix 1]

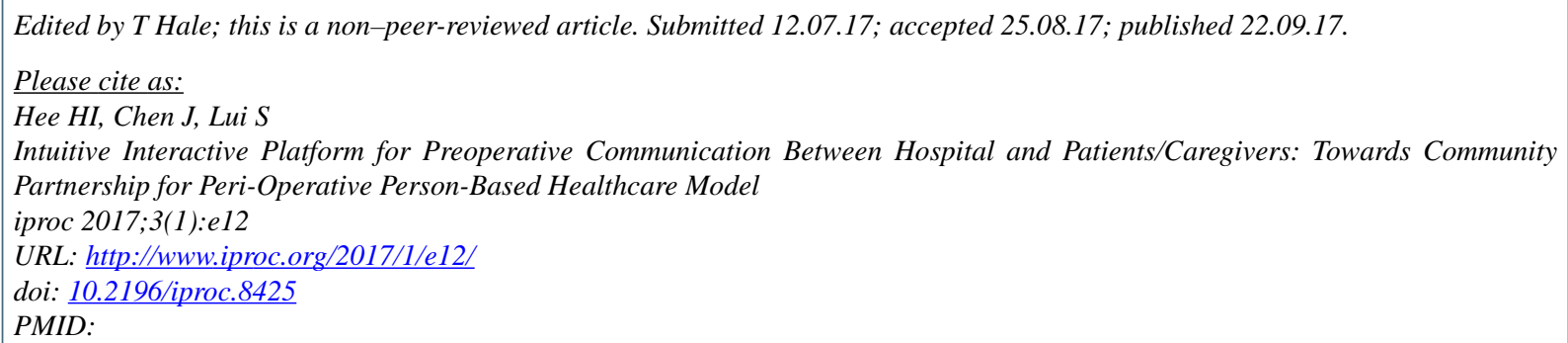

CHwan Ing Hee, Jacob Chen, Simon Lui. Originally published in Iproceedings (http://www.iproc.org), 22.09.2017. This is an open-access article distributed under the terms of the Creative Commons Attribution License (https://creativecommons.org/licenses/by/4.0/), which permits unrestricted use, distribution, and reproduction in any medium, provided the original work, first published in Iproceedings, is properly cited. The complete bibliographic information, a link to the original publication on http://www.iproc.org/, as well as this copyright and license information must be included. 